\title{
Testicular, Spermatic Cord, and Scrotal Soft Tissue Sarcomas: Treatment Outcomes and Patterns of Failure
}

\author{
Varun K. Chowdhry ${ }^{10},{ }^{1}$ John M. Kane III, ${ }^{2}$ Katy Wang, ${ }^{3}$ Daniel Joyce, ${ }^{4}$ \\ Anne Grand'Maison, ${ }^{5}$ and Gary N. Mann ${ }^{2}$ \\ ${ }^{1}$ Department of Radiation Medicine, Roswell Park Comprehensive Cancer Center, Buffalo, NY, USA \\ ${ }^{2}$ Department of Surgical Oncology, Roswell Park Comprehensive Cancer Center, Buffalo, NY, USA \\ ${ }^{3}$ Department of Biostatistics and Bioinformatic, Roswell Park Comprehensive Cancer Center, Buffalo, NY, USA \\ ${ }^{4}$ Department of Surgical Oncology, Cleveland Clinic, Cleveland, OH, USA \\ ${ }^{5}$ Department of Medicine, Roswell Park Comprehensive Cancer Center, Buffalo, NY, USA
}

Correspondence should be addressed to Varun K. Chowdhry; varun.chowdhry@roswellpark.org

Received 16 September 2020; Revised 15 January 2021; Accepted 9 February 2021; Published 5 March 2021

Academic Editor: Cornelis Verhoef

Copyright (c) 2021 Varun K. Chowdhry et al. This is an open access article distributed under the Creative Commons Attribution License, which permits unrestricted use, distribution, and reproduction in any medium, provided the original work is properly cited.

\begin{abstract}
Introduction. Paratesticular sarcomas are defined as tumors that arise within the scrotum and include the subsites of epididymis, spermatic cord, and tunica vaginalis and represent the most common type of GU sarcoma. The mainstay of treatment is often surgical resection, combined with histology specific chemotherapy and radiotherapy. Due to the rare nature of the disease, there are limited data to guide management. We present our single-institution retrospective experience regarding the management and treatment of paratesticular sarcomas. Materials and Methods. We queried our oncology registry database for patients treated for testicular, spermatic cord, and scrotal soft tissue sarcomas between 1971 and 2017. Patients in this series had pathological confirmation of a sarcoma diagnosis by a sarcoma-specialized pathologist. Only patients with localized disease were included in this analysis with the exception of patients with a diagnosis of rhabdomyosarcoma where patients with both localized and metastatic disease were included on this study. Results. A total of 34 patients were included in this retrospective analysis. The median was 24 (range, 5-78), and the median tumor size was $6.25 \mathrm{~cm}$. Twenty-six patients had localized disease $(76.6 \%)$ at the time of diagnosis. A predominance of patients had tumors involving the spermatic cord (45.5\%), and the most common histology was rhabdomyosarcoma (35.3\%), leiomyosarcoma (26.5\%), and well-differentiated liposarcoma (23.5\%). The median follow-up was 71.0 months (range, 2.5-534.4 months). A total of 7 patients experienced an isolated local failure (20.6\%), four patients developed distant metastatic disease (11.8\%), and one patient (2.9\%) with synovial sarcoma of the spermatic cord experienced a regional recurrence. The median progression-free survival (PFS) was 99.6 months, 95\% CI (45.8-534.3 months), with a three-year PFS rate of $71 \%, 95 \%$ CI (53\%-83\%), and a 5-year PFS rate of $64 \%$ (range, $46 \%-78 \%)$. We did not find any statistically significant associations based on surgery type $(p=0.15)$, the use of chemotherapy, $(p=0.36)$, or final margin status $(p=0.21)$. Two patients who were treated with preoperative radiotherapy had significant wound healing complication with chronic sinus tracts, though these patients did not experience a local recurrence. Conclusions. We provide a characterization of the natural history and treatment patterns of paratesticular sarcomas. While effective at reducing a local recurrence, preoperative radiotherapy was associated with significant toxicity. As a result, we prefer the use of postoperative radiotherapy in patients as clinically indicated. We did not find any specific treatment patterns associated with an improvement in clinical outcomes.
\end{abstract}




\section{Introduction}

Soft tissue sarcomas are an uncommon diagnostic entity, accounting for approximately $1 \%$ of all adult malignancies [1]. Paratesticular sarcomas account for an even rarer subset of soft tissue sarcomas, accounting for $1 \%$ of soft tissue sarcomas. However, paratesticular sarcomas are the most common presentation of genitourinary sarcomas, accounting for $30 \%$ of the overall cases [2].

Paratesticular sarcomas are defined as tumors that arise within the scrotum and include the epididymis, spermatic cord, and tunica vaginalis [3]. Patients will often present with scrotal swelling and a painless mass. Commonly, patients will undergo surgical resection without the clinical suspicion of sarcoma [4], and thus, consideration is given to additional surgery, chemotherapy, and radiotherapy. Much of the experience describing clinical patterns of behavior comes from small case series $[5,6]$ and have provided a framework to delineate pathways of treatment. Nevertheless, there is limited published experience on how to manage soft tissue sarcomas in this particular disease location. In general, principles of therapy from soft tissue sarcomas are employed, incorporating wide local resection, radiotherapy, and histologically driven systemic therapy as appropriate. However, the primary treatment modality for paratesticular sarcomas is considered to be surgical resection, with chemotherapy and radiotherapy utilized to facilitate surgical resection and decrease the local and distant recurrence [7]. Unlike the surgical approach for a primary testicular cancer which involves a radical inguinal orchiectomy with high dissection of the spermatic cord, surgery involves en bloc resection with negative margins.

In addition to oncologically oriented resection with negative margins, retroperitoneal lymph node dissection (RPLND) can be considered in patients either with retroperitoneal lymph node involvement or at having a high risk of nodal involvement. Here, we present our retrospective experience from a single institution regarding the management of testicular, spermatic cord, and scrotal soft tissue sarcomas.

\section{Methods}

After obtaining approval from our Institutional Review Board, our oncology registry database was retrospectively queried for patients treated for testicular, spermatic cord, and scrotal soft tissue sarcomas between 1971 and 2017. All patients in this series had pathological confirmation by a sarcoma-specialized pathologist either from a biopsy performed at our institution or secondary review of outside slides. For patients with rhabdomyosarcoma, patients with both localized and metastatic disease were included. For patients with other disease histologies, only patients with localized disease were included. Demographic, clinical, and pathological information was retrospectively recorded into a database. Survival curves were constructed using the Kaplan-Meier estimator method. Statistical Analysis was performed using SAS software (SAS v9.7; 100 SAS Campus Drive; Cary, NC 27513).

\section{Results}

A total of 34 patients were identified from our database. Demographic, clinical, and pathological information for patients in this series is shown in Table 1. The median age for patients in this series was 24 (range, 5-78), and the median tumor size was $6.25 \mathrm{~cm}$. Twenty-six patients had localized disease $(76.6 \%)$ at the time of diagnosis. Breakdown by tumor site and histology is reported in Table 1. A predominance of patients had tumors involving the spermatic cord $(45.5 \%)$, and the most common histology was rhabdomyosarcoma (35.3\%), leiomyosarcoma (26.5\%), and welldifferentiated liposarcoma (23.5\%). Eighty-five percent of patients had a biopsy/nononcologic resection in order to make a sarcoma diagnosis, including $73.5 \%$ via a transinguinal incision approach. Slightly more than one-third of patients underwent a formal sarcoma wide resection with en bloc removal of the tumor and surrounding tissues. Treatment characteristics are shown in Table 2.

The median follow-up was 71.0 months (range, 2.5-534.4 months). A total of 7 patients experienced an isolated local failure (20.6\%), four patients developed distant metastatic disease $(11.8 \%)$, and one patient $(2.9 \%)$ with synovial sarcoma of the spermatic cord experienced a regional recurrence in lymph nodes. No patient experienced a both a local and distant failure.

Of the seven patients who had local recurrences, we have detailed postrecurrence treatment information on four of those patients. Two of these patients had recurrence after an initial nononcological surgery, but had oncological re-excision and remained disease free. One patient had an asymptomatic local recurrence for a well-differentiated liposarcoma and chose no further therapy. The fourth patient who underwent reresection passed away during the immediate postoperative period.

Survival curves for progression-free survival and overall survival are shown in Figures 1 and 2. For all patients in the cohort, the median progression-free survival (PFS) was 99.6 months, 95\% CI (45.8-534.3 months), with a three-year PFS rate of $71 \%, 95 \%$ CI $(53 \%-83 \%)$, and a 5 -year PFS rate of $64 \%$ (range, 46\%-78\%). No local failures were noted in patients who received preoperative radiotherapy, but only two patients in this series were treated with preoperative radiation therapy for which follow-up data was available. However, both of these patients experienced significant wound healing issues with chronic sinus tracts.

We had at least 8 patients for the following histological subtypes: leiomyosarcoma, Rhabdomyosarcoma, and well-differentiated liposarcoma. PFS and OS are shown for these histological subtypes in Figures 3 and 4, respectively. It is noteworthy that patients with welldifferentiated liposarcoma had a 3-year progression-free survival of $100 \%$ and a five-year progression-free survival of $86 \%, 95 \%$ CI $(33 \%-98 \%)$. On the other hand, patients 
TABLE 1: Demographic information.

Total number of patients $(N)$

Median age at diagnosis (years)

34

Median tumor size $(\mathrm{cm})$

42 (range, 5-81)

6.25 (range, $0.8-14)$

$M$ stage at diagnosis

M0 $26(76.6)$

M1

$7(20.6 \%)$

Unknown

$1(2.9 \%)$

\section{Tumor site}

Scrotal

$11(33.3 \%)$

Spermatic cord

$15(45.5 \%)$

$8(21.2 \%)$

\section{Histology}

Well-differentiated liposarcoma

Dedifferentiated liposarcoma

$8(23.5 \%)$

Rhabdomyosarcoma

$1(2.9 \%)$

Embryonal rhabdomyosarcoma

$12(35.3 \%)$

Unknown type

$3(25 \%)$

$9(75 \%)$

Leiomyosarcoma

$9(26.5 \%)$

Myxoid liposarcoma

$2(5.9 \%)$

$2(5.9 \%)$

Synovial sarcoma

Method of diagnosis

Excisional biopsy/transcrotal surgery $\quad 14(41.2 \%)$

Inguinal orchiectomy

$18(52.9 \%)$

Hernia repair

$1(2.9 \%)$

Transcrotal surgery

$7(20.6 \%)$

No biopsy, definitive surgery only

$1(2.9 \%)$

Biopsy/nononcological resection prior to

surgery

Yes

$29(85.3 \%)$

No

$4(11.8 \%)$

Unknown

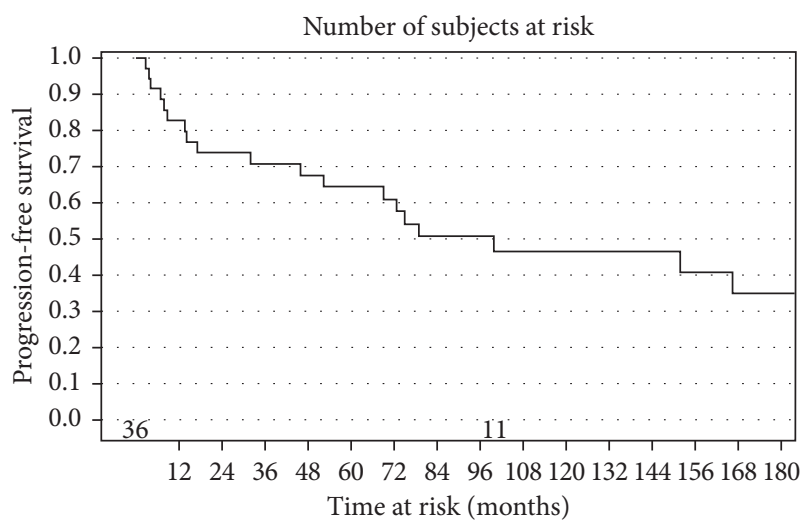

Figure 1: Kaplan-Meier curves for progression-free survival, all patients.

with rhabdomyosarcoma had a three-year progressionfree survival of $42 \%, 95 \%$ CI $(15 \%-67 \%)$.

The median overall survival (OS) was 99.6 months, $95 \%$ CI (45.8-534.3 months), with a three-year OS rate of $71 \%$, $95 \%$ CI $(53 \%-83 \%)$ and a 5 -year OS rate of $64 \%$ (range, $46 \%-78 \%)$. We did not find any statistically significant associations based on surgery type $(p=0.15)$, the use of chemotherapy, $(p=0.36)$, or final margin status $(p=0.21)$.

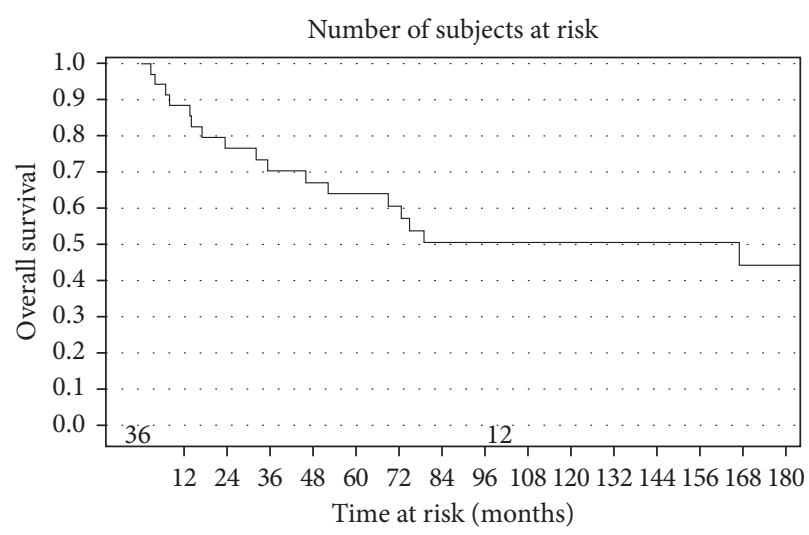

FIGURE 2: Kaplan-Meir curve for overall survival, all patients.

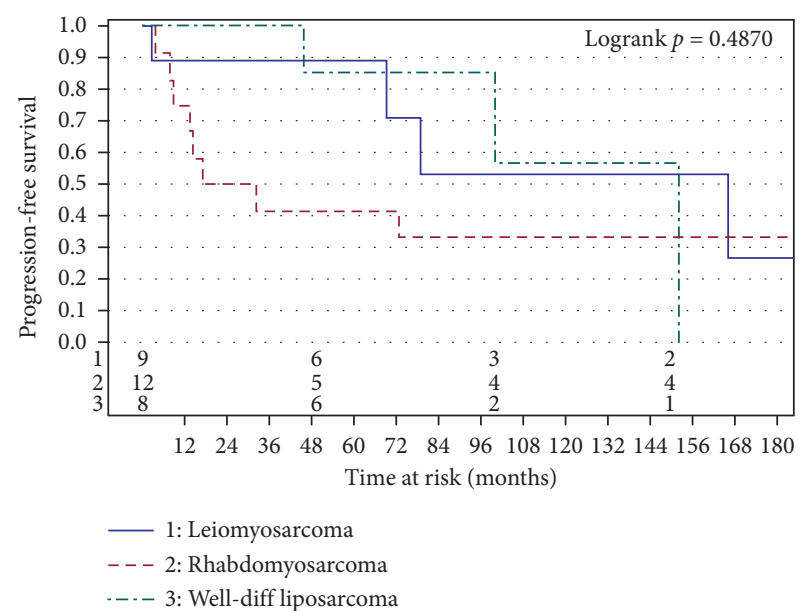

Figure 3: Progression-free survival by histology (for histologies, $n \geq 8$ ).

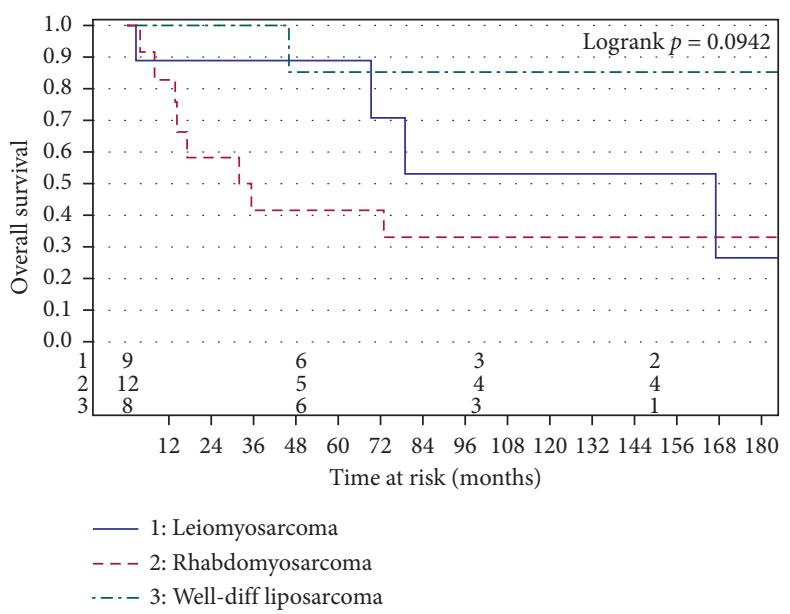

Figure 4: Overall free survival by histology (for histologies, $n \geq 8$ ).

\section{Discussion}

Adult paratesticular sarcomas represent a rare diagnostic entity, and often, initial management and surgical resection occur without clinical suspicion of sarcoma. Magnetic 
Resonance Imaging (MRI) can help delineate paratesticular tumors from tumors arising in the testis [8]. Core biopsy prior to undertaking definitive therapy can be useful, especially to guide appropriate preoperative therapy. Positronemission tomography (PET/CT) has been shown to be beneficial to evaluate nodal and distant metastatic staging in patients with rhabdomyosarcoma [9].

The surgical treatment of paratesticular sarcomas is challenging secondary to the close proximity to the reproductive organs, the abdominal wall/inguinal canal, and even the free intra-abdominal cavity. As with other soft tissue sarcomas, negative margin wide resection is the potentially curative treatment. The wide resection field needs to include any prior surgical biopsy site secondary to the potential for microscopic tumor seeding. In a series of pediatric patients with paratesticular rhabdomyosarcoma, scrotal violation has been shown to result in inferior outcomes, unless adequately addressed [10]. The conventional urology teaching for the biopsy of a possible testicular cancer is radical orchiectomy via an inguinal approach to prevent tumor seeding of the scrotum with possible secondary drainage to the inguinal lymph nodes. However, this algorithm is suboptimal for paratesticular sarcomas as an inguinal orchiectomy approach mandates subsequent en bloc resection of the entire inguinal skin/canal with a complex abdominal wall reconstruction. The need for full-thickness abdominal wall/inguinal canal resection also potentially exposes the free intra-abdominal cavity to microscopic tumor cells. Consequently, if there is a high suspicion for a paratesticular sarcoma (as opposed to testicular cancer), either image-guided trans-scrotal core needle biopsies or surgical biopsy via a trans-scrotal approach is preferred. An en bloc hemiscrotectomy to include all tissues at risk for microscopic tumor is more technically straightforward than abdominal wall resection/reconstruction. Limiting the tumor to the hemiscrotum also results in a more defined target for either preoperative or postoperative radiation.

When wide resection was not technically possible secondary to the extent of microscopic tumor seeding or patient comorbidities, strong consideration was given to adjuvant radiation therapy to improve local control. In contrast, preoperative radiation was often pursued when wide resection was being considered, but close or microscopically positive resection margins were anticipated. A preoperative radiation approach is standard for most extremity and truncal soft tissue sarcomas as there are both theoretical and clinical advantages as compared to postoperative radiotherapy. The therapeutic efficacy of preoperative radiation therapy is enhanced as compared with postoperative radiation therapy due to tumor oxygenation allowing the same therapeutic effect to be achieved at a lower radiation dose [11]. Additionally, treatment volumes are larger in the postoperative setting and include more normal tissue as the tumor will displace normal structures out of the radiation field in the preoperative setting. From the extremity sarcoma literature, preoperative radiotherapy has been associated with an increased risk of acute, reversible toxicity but with the benefit regarding the reduction in late toxicity $[12,13]$.
Although neither of the patients who received preoperative radiation therapy experienced a local recurrence in our series, both patients developed postoperative wound healing issues with chronic sinus tracts. Therefore, our institutional preference for paratesticular sarcomas undergoing wide resection has actually changed over time to favor postoperative radiation once the surgical site has completely healed, especially given the potential morbidity of groin radiation due to skin folds, high bacterial counts, significant moisture, etc.

The use of neoadjuvant and adjuvant chemotherapy in sarcomas has always been a source of debate and remains controversial, given the lack of strong data. As a result, the patients in this series who received chemotherapy were largely patients with RMS and were treated with a vincristine-, adriamycin-, and cyclophosphamide-based regimens. Neoadjuvant chemotherapy has several advantages including downsizing the tumor, allowing less extensive surgical excision, and testing chemotherapy sensitivity but most importantly, treating micrometastases and potentially reducing the risk of developing metastatic disease. No randomized placebocontrolled trial has yet confirmed a survival benefit from neoadjuvant chemotherapy. Nevertheless, many centers are using preoperative chemotherapy in patients at high risk of developing metastatic disease based on sarcoma histopathology, tumor size, and grade. The use of neoadjuvant chemotherapy in sarcoma remains institution dependent. With the exception of rhabdomyosarcoma, neoadjuvant chemotherapy was not largely used during the period this cohort of patients was treated, therefore limiting any conclusion about the efficacy of neoadjuvant chemotherapy in paratesticular sarcomas. The role of adjuvant chemotherapy in sarcoma has been studied more extensively. The strongest evidence of survival benefit comes from the meta-analysis published by Pervaiz et al. [14] who demonstrated a $11 \%$ absolute risk reduction of death. Since only $11.7 \%$ of patients in our cohort received adjuvant chemotherapy, our data are insufficient to draw a meaningful conclusion about the benefit of adjuvant chemotherapy in paratesticular sarcomas.

We will now address some of the limitations of our paper. As a retrospective analysis, it is difficult to make any definitive conclusions regarding treatment pathways that could be best for patients. As a tertiary referral center, many patients came from the surrounding community, and often, we only have follow-up data regarding surgical outcomes, while other variables, including chemotherapy and radiotherapy details, are missing. Additionally, given the small number of patients, combined with several different histopathologies, we did not find any specific disease- or treatment-related factors that were definitively associated with improved outcomes. It is also important to note that while as a practice, we centrally review of pathology by a sarcoma-trained histopathologist, we did have limited ability to capture more specific histopathological information for patients included from earlier time points of the database. For example, we were unable to capture 
TABLE 2: Treatment.

\begin{tabular}{|c|c|}
\hline \multicolumn{2}{|l|}{$\begin{array}{l}\text { Type of definitive surgery } \\
\text { Local surgery }\end{array}$} \\
\hline No local surgery* & $12(35.3 \%)$ \\
\hline WLE, en bloc removal & $13(38.2 \%)$ \\
\hline Inguinal orchiectomy & $7(20.6 \%)$ \\
\hline Missing & $2(5.9 \%)$ \\
\hline \multicolumn{2}{|l|}{ Nodal dissection } \\
\hline Retroperitoneal node dissection & $5(14.7 \%)$ \\
\hline \multicolumn{2}{|l|}{ Margin status } \\
\hline Positive & $3(8.8 \%)$ \\
\hline Negative & $11(34.4 \%)$ \\
\hline Close $(<1 \mathrm{~mm})$ & $2(5.9 \%)$ \\
\hline Unknown margin status & $18(52.9 \%)$ \\
\hline \multicolumn{2}{|l|}{ Chemotherapy } \\
\hline Adjuvant & $4(11.7 \%)$ \\
\hline Therapeutic & $6(17.6 \%)$ \\
\hline \multicolumn{2}{|l|}{ Radiotherapy** } \\
\hline Any radiation therapy & $7(34 \%)$ \\
\hline Preoperative & $3(8.8 \%)$ \\
\hline Postoperative & $4(11.8)$ \\
\hline Mean preoperative radiation dose $\mathrm{e}^{* * *}$ & 5220 cGy (range, 5000-5040 cGy) \\
\hline Whole abdomen radiation dose (RMS only) & 1805 cGy \\
\hline
\end{tabular}

specific types of rhabdomyosarcoma for some patients as a result.

It is noteworthy that our calculated progression-free survival and overall survival are nearly identical, as for many patients, the most recent follow-up we have is the date of death. We found a median overall survival (OS) of 99.6 months, 95\% CI (45.8-534.3 months), with a three-year OS rate of $71 \%$ and a 5 -year OS rate of $64 \%$. Our results are consistent with the experience at the Memorial Sloan Kettering Cancer Center, who reported a five-year disease-free survival of $75 \%$ [15].

We did not demonstrate differences in clinical outcome by surgery type. Although we believe in the importance in en bloc excision for selected patients, there are several variables that can confound the importance of surgical excision with widely negative margins. With the exception of patients with well-differentiated liposarcomas, patients with scrotal sarcomas are at high risk of both local and distant recurrence. Therefore, the potential benefits of radiation and systemic chemotherapy must be reconciled with the limited benefits of an extensive, technically complex, morbid wide resection that could otherwise delay systemic therapy. Furthermore, the number of patients in this series may be too small to stratify by other important variables such as histology, tumor size, and grade. Additionally, patients were offered treatment based on pretreatment and disease-specific associated risk factors. As this is an uncontrolled series, the diverse histologies likely impacted the treatment a patient would have received. For example, a patient with a small well-differentiated liposarcoma may have a favorable disease course even without further treatment. On the other hand, a patient with large, high-grade tumor was offered more aggressive therapies, making it difficult to analyze the efficacy of the particular therapy. Additionally, over one-third of the patients in this series had a diagnosis of a rhabdomyosarcoma, where the mainstay of treatment involves systemic chemotherapy and radiotherapy. Therefore, aggressive surgical resection may provide little added benefit due to the high rates of local control that can be achieved with other therapies.

The role of RPLND in the management of scrotal and paratesticular soft tissue sarcomas is considered controversial [16]. Catton et al. reported that patients presenting with primary disease had a regional recurrence rate of $28 \%$ suggesting that patients with rhabdomyosarcoma, fibrous histiocytoma, or fibrosarcoma may require RPLND [17]. Our data suggest RPLND is not needed for most patients with paratesticular sarcomas. We only had one patient (2.9\%) with an isolated regional failure in the inguinal lymph nodes in a patient diagnosed with synovial sarcoma on the spermatic cord. Regional nodal recurrence rates are uncommon in extremity soft tissue sarcomas [18], and our data also suggest that regional lymph node recurrence of paratesticular soft tissue sarcomas is rare as well. The low rate of nodal relapse in our series is consistent with other published reports [19]. As a result, our data suggest that routine lymph node dissection and radiotherapy to regional lymphatics is not indicated for most paratesticular soft tissue sarcomas. The exception of this is patients with paratesticular rhabdomyosarcoma benefit from RPLND, where the available data suggest that imaging incorrectly stages less than half of the patients, and thus, surgical ipsilateral retroperitonal 
lymph node dissection is considered to be the standard of care [20].

\section{Conclusions}

We describe our treatment approach and philosophy regarding the management of paratesticular and scrotal soft tissue sarcomas, as well as outcomes of patients treated at our institution. Although our series does not definitely demonstrate the importance of oncological surgery with widely negative margins, we agree with the guidelines from the National Comprehensive Cancer Network (NCCN) that obtaining oncologically appropriate margins is important for most patients, with consideration of radiation therapy and chemotherapy in selected patients based on specific type [21]. Due to significant toxicity experienced in patients treated preoperative radiation therapy, we favor postoperative radiation therapy once all surgical wounds have healed. Finally, neoadjuvant chemotherapy should be considered in high-grade histology to reduce the risk of developing metastatic disease and improve outcome based on pathologically driven treatment paradigms for sarcomas that occur in different anatomic subsites.

\section{Data Availability}

The data used to support the findings of this study are available from the corresponding author upon request.

\section{Ethical Approval}

This study was approved by the Institutional Review Board.

\section{Conflicts of Interest}

The authors report no conflicts of interest with this work.

\section{References}

[1] R. L. Siegel, K. D. Miller, and A. Jemal, "Cancer statistics, 2020,” CA: A Cancer Journal for Clinicians, vol. 70, no. 1, pp. 7-30, 2020.

[2] F. Gomez-Rivera, T. D. Shellenberger, and E. M. Sturgis, "Genitourinary sarcomas," in Sarcoma Oncology: Multidisciplinary Approach, R. E. Rollock, R. L. Randall, and B. O’Sullivan, Eds., pp. 229-230, PMPHUA, Raleigh, NC, USA, 2019.

[3] R. A. Keenan, A. U. Nic An Riogh, A. Stroiescu et al., "Paratesticular sarcomas: a case series and literature review," Therapeutic Advances in Urology, vol. 11, Article ID $175628721881802,2019$.

[4] B. Khoubehi, V. Mishra, M. Ali, H. Motiwala, and O. Karim, "Adult paratesticular tumours," BJU International, vol. 90, no. 7, pp. 707-715, 2002.

[5] U. Küçük, U. Bayol, E. E. Pala, O. Akman, and R. T. Divrik, "Paratesticular sarcomas: our case series," Türk Üroloji Dergisi/Turkish Journal of Urology, vol. 39, no. 2, pp. 84-89, 2013.

[6] Y. Ünlü, G. E. Huq, G. Özyalvaçli et al., "Paratesticular sarcomas: a report of seven cases," Oncology Letters, vol. 9, no. 1, pp. 308-312, 2015.

[7] P. G. Casali, N. Abecassis, H. T. Aro et al., "Soft tissue and visceral sarcomas: ESMO-EURACAN clinical practice guidelines for diagnosis, treatment and follow-up," Annals of Oncology, vol. 29, no. 4, pp. 268-269, 2018.

[8] P. J. Woodward, C. M. Schwab, and I. A. Sesterhenn, "From the archives of the AFIP," Radiographics, vol. 23, no. 1, pp. 215-240, 2003.

[9] U. Tateishi, A. Hosono, A. Makimoto et al., "Comparative study of FDG PET/CT and conventional imaging in the staging of rhabdomyosarcoma," Annals of Nuclear Medicine, vol. 23, no. 2, pp. 155-161, 2009.

[10] W. J. Hammond, B. A. Farber, A. P. Price et al., "Paratesticular rhabdomyosarcoma: importance of initial therapy," Journal of Pediatric Surgery, vol. 52, no. 2, pp. 304-308, 2017.

[11] A. Kaushal and D. Citrin, "The role of radiation therapy in the management of sarcomas," Surgical Clinics of North America, vol. 88, no. 3, pp. 629-646, 2008.

[12] B. O’Sullivan, A. M. Davis, R. Turcotte et al., "Preoperative versus postoperative radiotherapy in soft-tissue sarcoma of the limbs: a randomised trial," The Lancet, vol. 359, no. 9325, pp. 2235-2241, 2002.

[13] A. Davis, B. Osullivan, R. Turcotte et al., "Late radiation morbidity following randomization to preoperative versus postoperative radiotherapy in extremity soft tissue sarcoma," Radiotherapy and Oncology, vol. 75, no. 1, pp. 48-53, 2005.

[14] N. Pervaiz, N. Colterjohn, F. Farrokhyar, R. Tozer, A. Figueredo, and M. Ghert, "A systematic meta-analysis of randomized controlled trials of adjuvant chemotherapy for localized resectable soft-tissue sarcoma," Cancer, vol. 113, no. 3, pp. 573-581, 2008.

[15] J. Coleman, M. F. Brennan, K. Alektiar, and P. Russo, "Adult spermatic cord sarcomas: management and results," Annals of Surgical Oncology, vol. 10, no. 6, pp. 669-675, 2003.

[16] D. Bansal, J. Varghese, M. Babu et al., "Leiomyosarcoma presenting as a scrotal mass," Urology Case Reports, vol. 7, pp. 42-44, 2016.

[17] C. N. Catton, B. J. Cummings, V. Fornasier, B. O’Sullivan, I. Quirt, and D. Warr, "Adult paratesticular sarcomas: a review of 21 cases," Journal of Urology, vol. 146, no. 2, pp. 342-345, 1991.

[18] S. Riad, A. M. Griffin, B. Liberman et al., "Lymph node metastasis in soft tissue sarcoma in an extremity," Clinical Orthopaedics and Related Research, vol. 426, pp. 129-134, 2004.

[19] M. T. Ballo, G. K. Zagars, P. W. T. Pisters, B. W. Feig, S. R. Patel, and A. C. v. Eschenbach, "Spermatic cord sarcoma: outcome, patterns of failure and management," Journal of Urology, vol. 166, no. 4, pp. 1306-1310, 2001.

[20] B. P. Hermans, R. S. Foster, R. Bihrle et al., "Is retroperitoneal lymph node dissection necessary for adult paratesticular rhabdomyosarcoma?" Journal of Urology, vol. 160, no. 6, pp. 2074-2077, 1998.

[21] NCCN, NCCN Clinical Practice Guidelines in Oncology. Soft Tissue Sarcoma. NCCN Evidence Blocks. Version 2, NCCN, Philadelphia, PA, USA, 2020, https://www.nccn.org/ professionals/physician_gls/pdf/sarcoma_blocks.pdf. 